\title{
La Paranoia Benigna. Recordando a los Clásicos
}

\author{
María del Prado Ordóñez Fernándeza
}

a F.E.A. de Psiquiatría del Hospital General de Ciudad Real.

Correspondencia: $\mathrm{M}^{\mathrm{a}}$ del Prado Ordóñez Fernández, C/ Alarcos $\mathrm{n}^{\circ} 3,1^{\circ} \mathrm{D}, 13001$ - Ciudad Real, España. E-mail: mpordonez@ sescam.jccm.es

Recibido el 20 de diciembre de 2010.

Aceptado para su publicación el 28 de febrero de 2011.

\section{RESUMEN}

Entre los pacientes que acuden a las consultas de Salud Mental con el diagnóstico de trastorno delirante se encuentra un subgrupo integrado por trastornos delirantes, generalmente del tipo alusivo-referencial, que ceden con el tratamiento, sin ameritar ingreso en la mayoría de las ocasiones, con una personalidad de base capaz de introspección y autocrítica, con confianza en el médico, actitud que fomenta la alianza terapéutica y la adherencia al tratamiento. Estos trastornos delirantes tienen una evolución favorable.

El objetivo de este artículo es repasar la evolución histórica de la paranoia benigna, con la finalidad de comprender mejor la patología psiquiátrica de este subgrupo curable.

Palabras clave. Trastornos Paranoides.

\section{ABSTRACT}

Benign paranoia. Remembering the Classics

Amongst patients diagnosed with delirium disorder attending Mental Health clinics there is a subgroup of patients with delirium disorders, mainly of the allusive-referential type, who respond to treatment, without having to be hospitalised. They are capable of introspection and self-criticism. They have confidence in their doctor which encourages a therapeutic alliance and treatment adherence. These delirium disorders have a favourable evolution.

The objective of this article is to review the history of benign paranoia with the aim of gaining a better understanding of the psychiatric disease of this curable subgroup.

Key words. Paranoid Disorders.

\section{HISTORIA}

En 1892 Wernicke cita en su obra Zirkumskripte Autopsychose aufgrund einer überwertigen Idee ${ }^{1}$ el caso de una señorita de 40 años de edad, profesora de un instituto superior de enseñanza femenina, que se había enamorado secretamente de un colega. Ella creía notar que él correspondía a su pasión. En todo momento pensaba, convencida, en ello, y le parecía que hasta podía confirmar sus observaciones con los más nimios detalles cotidianos. Al final esperó a que él se le declarase algún día, pero llegó un momento en que dicho profesor se marchó del lugar, sin sospechar nada de los sentimientos de ella. En relación con esta vivencia amorosa interior, se desarrolló en ella un delirio de referencia sistematizado: las alumnas, las colegas y los demás profesores conocían la situación, se comportaban de una manera diferente, hacían observaciones alusivas, y hasta el director se había inmiscuido, con toda seguridad, en el asunto. Al final, la profesora estaba firmemente convencida de que las intrigas del profesorado habían destruido sus esperanzas con respecto al joven profesor. Wernicke, que tuvo ocasión de seguir observándola en su vida profesional muchos años después de la afección, la describe como una persona muy culta y sensible, de conceptos muy delicados en lo que atañe al honor y al deber, con una vida afectiva muy excitable interiormente, y en su relación amorosa distanciada y hasta áspera. Era una profesora muy apta y laboriosa, que seguramente había trabajado en exceso. Con esta descripción ilustra Wernicke lo que llama autopsicosis circunscrita basada en una idea sobrevalorada. 
Este autor ha comprobado que existen formas de delirio de referencia circunscrito, que surgen directamente de una vivencia real, y que contienen su núcleo en dicha vivencia. Wernicke cree que estas vivencias, que muchas veces no faltan ni en la vida psíquica normal y sana, conducen al delirio de referencia a causa de la intensidad del afecto que las acompaña1.

En 1905 Friedmann llama la atención sobre cierto número de casos en los que el delirio aparece muy claramente como reacción a una vivencia determinada, con una evolución relativamente favorable. A estos casos los designa con el nombre de paranoia benigna, indicando tres rasgos de carácter propios de tales sujetos: sensitivos, tenaces, exaltados ${ }^{2}$.

En 1909 Robert Gaupp da el nombre de paranoia abortiva a ciertos delirios de persecución que pueden sanar. Según Gaupp se trata de hombres instruidos, cuya edad está entre los 25 y los 45 años, que se han mostrado durante toda la vida de humor benévolo, modestos, poco seguros de sí mismos, un tanto ansiosos, muy concienzudos, escrupulosos incluso, hombres, en una palabra, que por toda su manera de ser se nos muestran emparentados con los enfermos que sufren obsesiones. Naturalezas reflexivas, inclinadas a la autocrítica, seres sin ninguna sobrestimación de sí mismos, sin humor combativo. En ellos se instala de una manera completamente insidiosa, en la mayoría de los casos, con un vínculo temporal más o menos estrecho con una vivencia de fuerte carga afectiva, un sentimiento de inquietud ansiosa con ideas de persecución. Junto con esto se da en ellos cierta conciencia de la enfermedad psíquica y se quejan de síntomas psicasténicos.

Estos seres, cuya naturaleza es moralmente delicada, se ponen a pensar, por principio de cuentas, si sus enemigos no tendrán efectivamente razón al pensar mal de ellos, si incluso ellos mismos, por su conducta, no habrán dado ocasión para una crítica maligna o para una intervención de la policía, o hasta para un juicio en los tribunales. Pero no se manifiesta ningún estado melancólico, ningún delirio de autoacusación. Aparecen, por el contrario, ideas de persecución de un significado cada vez más y más preciso, coherentes, bien fundadas lógicamente, y que van orientadas contra personas o contra determinados organismos profesionales (la policía, etc.). El delirio de relación no se extiende a todo el círculo que rodea al enfermo. Así, por ejemplo, el médico mismo nunca será incluido en la formación delirante durante una permanencia de varios meses en la clínica.

El enfermo, por el contrario, experimenta cierta necesidad del médico, porque la seguridad de que ningún peligro lo amenaza y de que en la clínica le están garantizando la ayuda y la protección actúa algunas veces sobre él de manera apaciguadora. Una charla seria con el médico puede aliviarlo durante cierto tiempo, pero seguramente no en forma duradera. Hacen a veces algunas concesiones y admiten que se trata de una desconfianza patológica, de una asociación particular mórbida, pero nuevas percepciones en el sentido del delirio de interpretación aportan entonces precisamente un nuevo material al sistema de persecución. Con el progreso de la afección ansiosa, teñida de desconfianza, que evoluciona a lo largo de grandes oscilaciones, las ideas de persecución se van haciendo más precisas, y ocasionales ilusiones sensoriales refuerzan el sentimiento de su realidad. En momentos más tranquilos se muestra cierta lucidez sobre las ideas de persecución anteriores: "Evidentemente, eso es entonces algo que he imaginado". Así prosigue la enfermedad durante años, cediendo unas veces, exacerbándose otras. Subsiste siempre el fondo de humor de pusilanimidad ansiosa y el enfermo está dominado por esta reflexión: “¿Qué he hecho para merecer esas señales de hostilidad?" Si alguna vez llega a revelarse contra esa tortura perdurable, o incluso a defenderse contra la agresión delirante, es sólo de manera pasajera. Nunca hay en estos enfermos actitudes altivas ni orgullo, nunca hay ideas de grandeza, ninguna huella de debilidad mental, sino, al contrario, una conducta del todo natural. Los enfermos que vienen libremente a la clínica y que salen de ella cuando bien les parece tienen hasta el fin toda su confianza en el médico, y se complacen en regresar para consultarlo cuando, en la práctica de su profesión, se sienten de nuevo más perseguidos e importunados. Vienen entonces con esta pregunta: ¿Es posible que esto no sea realmente más que cosa de la imaginación? Lo más frecuente es que no se observe ninguna progresión clara de la enfermedad, aunque esto no siempre sea así.

En uno de los casos observados las asociaciones mórbidas típicas existen desde hace doce años, y sin embargo no ha llegado a constituirse ningún sistema delirante rígido. Se trata más bien de ideas de persecución que varían en su fuerza. Con todo eso, el enfermo es capaz de desempeñar la profesión en que está ocupado. En períodos 
relativamente buenos no deja de hacerse sentir una semiconciencia de la enfermedad. La idea prevalente no domina al sujeto en su totalidad, o sea en la medida en que lo hace en el delirio de reivindicación. En todos los casos la disposición depresiva escrupulosa existía desde siempre $^{3}$.

Gracias a la introducción de estos casos se amplía el marco de la paranoia, ensanchándose a su vez, también, el campo de los mecanismos implicados en la misma. Para Friedmann, los factores implicados son vivenciales, mientras que Gaupp insiste en su origen caracterógeno ${ }^{4}$.

Muchos de estos pacientes, de evolución benigna, no eran tratados en asilos, sino en consultorios particulares ${ }^{4}$.

Kraepelin, en su edición de 1915, admite casos curables en el cuadro por él descrito como paranoia: "no se puede oponer ninguna objeción fundamental a la producción de una paranoia benigna, psicógena, con camino abierto hacia la curación"

En un estudio notable, publicado en 1924, Lange hace una especie de repaso general de los casos clínicos diagnosticados de paranoia, concluyendo que la paranoia crónica tipo Kraepelin era sumamente rara, admitiendo las formas curables o benignas ${ }^{6}$.

Entre los delirios paranoicos, Kretschmer se propone aislar "un grupo absolutamente caracterizado por sus causas, su forma y su evolución". A este grupo le da el nombre de Delirio de relación de los sensitivos ${ }^{7}$.

\section{DELIRIO SENSITIVO DE REFERENCIA DE KRETSCHMER}

\section{Causas}

Aunque Kretschmer no deja de admitir una base biológica para estas psicosis, considera que las causas determinantes del delirio están constituidas por la combinación de tres elementos: carácter, vivencia y medio social ${ }^{7}$.

El carácter responde al tipo designado por Kretschmer con el término sensitivo. De él toma su nombre el delirio descrito ${ }^{4}$. El carácter sensitivo, nos dice Krestchmer, no tiene nada de un estado innato y fijo, de un estado constitucional: es una disposición adquirida a lo largo de la evolución, y en la que tienen el papel principal ciertos traumas afectivos determinantes ${ }^{4}$.

El tipo sensitivo que nos ocupa es definido a partir de reacciones propias frente a acontecimientos de fuerte carga afectiva: esta reacción en el orden del comportamiento se distingue por una gran capacidad de impresión ante los acontecimientos vitales, con excitabilidad e hipersensibilidad y por una falta de conducción que detiene la descarga por la acción. A esta detención corresponde la contención (Verhaultung) en la conciencia de las representaciones correspondientes. La energía psíquica movilizada por la vivencia se retiene, acumulándose alrededor del grupo emotivo, el cual se convierte enseguida en un cuerpo extraño torturante, sentido conscientemente en el interior de la consciencia. Por lo tanto, esta contención no es sino una exageración de la función de retención (Retention) de los complejos ideo-afectivos. La representación del acontecimiento y el estado afectivo desagradable que con ella va ligado tienden a reproducirse indefinidamente en la conciencia. Este modo reaccional de la contención es, así, todo lo contrario de la "represión" (refoulement) que en la histeria, por ejemplo, relega al inconsciente el "recuerdo" penoso".

Si los estados afectivos se clasifican en esténicos y asténicos según su intensidad, su duración y su capacidad de exteriorización, en los sensitivos se puede comprobar una curiosa mezcla de tendencias esténicas (elevada impresionabilidad ante los acontecimientos adversos) y asténicos (dificultad de exteriorización, falta de conducción, con retención y contención). Estas últimas son las que dominan, pero al precio de una viva tensión producida por la sobrestimación esténica de los fracasos, de orden ético. Esta tensión es la que constituye el factor psicológico determinante en los delirantes sensitivos, los cuales, en suma, están completamente subyugados por las tensiones sociales y éticas ${ }^{7}$.

El conflicto central, en estos sujetos, está formado en efecto por el sentimiento que experimentan de su inferioridad en el orden ético, sentimiento que viene a ser reavivado por cada fracaso vital y que es reanimado sin cesar en la conciencia por la contención. De ello resulta una exaltación puramente reaccional del amor propio, completamente distinta de la exaltación primaria del amor propio en el sujeto esténico. Al contrario que en el esténico, en el sensitivo la autoestima, más que excesiva, es continuamente cuestionada aunque deseada como excesiva $^{7}$. 
En la pintura que Kretschmer hace de estos sujetos de tipo sensitivo vemos que les da, por una parte, una extraordinaria impresionabilidad, una sensibilidad sumamente accesible y vulnerable, pero también, por otra parte, cierta dosis consciente de ambición y de tenacidad. Los representantes acabados de este tipo son personalidades complicadas, muy inteligentes, de valor muy alto, de sensibilidad fina y profunda, de una ética escrupulosa, y que en los casos del corazón son de una delicadeza excesiva y de un ardor completamente interiorizado. Son víctimas predestinadas de todas las durezas de la vida. Mantienen en sí mismos profundamente encerradas la constancia y la tensión de sus sentimientos. Poseen capacidades refinadas de introspección y de autocrítica. Son muy susceptibles y tercos, pero, al mismo tiempo, particularmente capaces también de amor y de confianza. Se tienen a sí mismos en justo aprecio, y sin embargo son tímidos y están llenos de inseguridad cuando se trata de producir algo suyo. Vueltos hacia sí mismos y sin embargo abiertos y filántropos, modestos, pero de una voluntad ambiciosa, poseen, por lo demás, altas virtudes sociales. Lo que se desprende muy claramente de esta descripción es que el carácter sensitivo no puede considerarse como una disposición constitucional o afectiva simple, sino que representa una personalidad en toda su complejidad $^{4}$.

Pero este carácter sensitivo puro no es tan fácil de encontrar en la clínica, y el mismo Kretschmer, en el capítulo séptimo de su obra El Delirio sensitivo de referencia, habla de variantes carectorológicas, que se pueden dar en una misma personalidad, mezclas de rasgos sensitivos con rasgos de otras formas caracterológicas (primitivo, expansivo, histérico). Esta combinación de rasgos hace variar los cuadros patológicos hacia formas más persecutorias o más combativas o combinaciones sensitivas-desiderativas, sensitivas-persecutorias, etc. "Cuanto más sensitivo es un carácter, tanto más específicamente reaccionará ante un complejo de culpabilidad con un delirio de relación de estructura fina"?.

El segundo elemento descrito por Kretschmer en la etiología de las psicosis es un determinado acontecimiento: un acontecimiento esencialmente caracterizado por el modo como es vivido, y es eso lo que expresa directamente el término alemán Erlebnis ("vivencia"). La vivencia (grupo de sensaciones y de representaciones susceptibles de producir afecto) es aquella que le revela al sujeto su propia "insuficiencia", aquella que "lo humilla": el sentimiento de fracaso".
El tercer factor etiológico es el medio social. El medio actúa sobre la manifestación de la enfermedad "según una fórmula única: tensión del amor propio en una situación oprimente". Esta "vivencia es el conflicto externo del hombre aislado e incapaz ante las ordenaciones rígidas y omnipotentes de la sociedad, con sus leyes, con su disciplina, con su opinión pública: la vivencia de la violación de la voluntad individual por la voluntad colectiva"?

\section{Forma}

El delirio sensitivo descrito por Kretschmer es más hiposténico y menos agresivo, ya que el carácter que lo sostiene es menos rígido. Es un delirio alusivo, refencial: alusiones peyorativas y malintencionadas procedentes de los familiares, superiores jerárquicos o colegas del trabajo.

El carácter sensitivo por él descrito se corresponde con sujetos tímidos, sensibles, a menudo ansiosos y psicoasténicos (escrúpulos, vacilaciones, etc.), que se sienten particularmente inclinados a las luchas de conciencia y muy sensibles a las reacciones de los demás (hiperestesia de los contactos sociales). Estos sujetos inhiben fuertemente sus pulsiones, estando profundamente insatisfechos. En ellos los complejos de frustración y de inferioridad son manifiestos y es, sobre este terreno, sobre este fondo de sensibilidad, fácilmente impresionable y vulnerable, cómo el cúmulo de circunstancias penosas, la suma de fracasos o de conflictos, la tensión engendrada por la exasperación, por las decepciones o por la desesperación, desencadenan la psicosis ${ }^{8}$. Los conflictos en estos sujetos conducen a una tensión afectiva cada vez mayor, por sus dificultades para conducir los afectos retenidos. A veces, una gota de agua hace desbordar el vaso y es a raíz de una discusión, de un avatar o de una humillación, cuando estalla el delirio, desencadenándose la transformación del contenido vivencial primario en un grupo de representaciones secundario, patológico y aparentemente extraño, que desde ese momento representa un mecanismo psíquico independiente, separado de dicho contenido. A este proceso patológico que representa el grado máximo de retención Kretschmer lo denomina inversión, entendiendo por ésta la transformación refleja de un grupo de representaciones, conscientes y exageradamente elaboradas en el interior, hasta constituir un mecanismo de pensamiento secundario (una vivencia simbólica secundaria), independiente y consciente, relacionado asociativamente con la vivencia primaria, y basado en ideas delirantes de referencia o alusión $n^{7}$. 
Sobre la semiología Kretschmer escribe": "El núcleo del cuadro mórbido es un delirio de relación concéntrico, fundado sobre una base afectiva que presenta todos los grados, de la seguridad humillante a la autoacusación, experimentada hasta la desesperación". Toda la semiología se concentra en tres motivos:

1. El contenido representativo y el estado afectivo están absolutamente centrados, durante el período de estado de la enfermedad, en torno a la experiencia patógena.

2. Lossíntomas delapsicosis sensitivarepresentan el efecto exaltado de las propiedades del carácter sensitivo.

3. El cuadro mórbido suele estar coloreado de síntomas de agotamiento.

Veamos cómo desarrolla Kretschmer esos tres puntos ${ }^{4}$ :

1. "La experiencia decisiva, con la situación vital que subyace a ella, lo es simplemente todo. Si la quitamos, la enfermedad quedará reducida a nada. Con su repetición en la obsesión, la vivencia constituye el objeto siempre nuevo de los remordimientos, de los miedos hipocondríacos..., de los accesos de ansiedad y de desesperación, de los vanos esfuerzos de la voluntad; es ella la fuente del humor y la meta de los pensamientos".

2. "Todos los rasgos de la personalidad sensitiva reaparecen, exagerados, en el delirio, y explican los contenidos mismos del delirio, las oscilaciones de la convicción delirante (vaivenes entre la representación obsesiva y la convicción delirante), la intensidad afectiva de los paroxismos, la ausencia ordinaria de reacciones agresivas, su carácter únicamente defensivo en los casos puros, el acento hipocondríaco del cuadro, el esfuerzo hacia el restablecimiento y la confianza con que se acude al médico".

3. "El estado nervioso de agotamiento psíquico, finalmente, da al cuadro unas características completamente distintas, reflejando el estado de estos sujetos que a menudo durante años han mantenido en el extremo de la tensión sus débiles fuerzas para atormentarse a sí mismos con sus conflictos".

El delirio de relación sistemático, con conservación de la lógica y de la reflexión, no es descrito por Kretschmer más que como la forma sintomática más frecuente, si no la más típica, de la relación delirante del sensitivo. El autor llama la atención "sobre la masa enorme de las ideas de relación, que son de una abundancia sin otro ejemplo, y sobre la delicadeza de sus ramificaciones, sobre el espíritu de combinación que nunca se harta de construir las correspondencias más ingeniosas a propósito de conversaciones de la más cotidiana trivialidad, de artículos de periódico, de la profesión y de los ires y venires de los vecinos, de un roce de ropa, de una puerta que se abre, de un ruido de la calefacción, etc."

Pero, al lado de esta forma típica, el autor distingue otras tres formas de psicosis sensitivas. La primera de ellas es la confusión aguda sensitiva (akuter dissoziativer Wahnsinn), que aparece como una etapa crítica de corta duración y responde a los casos más graves de psicosis sensitiva. Este Wahnsinn agudo se manifiesta por esbozos de disociación psíquica, "es decir, por síntomas intelectuales emparentados con la catatonia y con la esquizofrenia, como por ejemplo sentimientos de influencia, de acción a distancia, de transmisión del pensamiento y de la extrañeza, por un relajamiento de las asociaciones, y por tendencias a pasar al delirio de grandeza". El diagnóstico puede ser difícil si hay un acceso evolutivo esquizofrénico verdadero ${ }^{4}$.

Las otras dos formas son la racha delirante emparentada con el tipo neurótico obsesional (sprunghafte Wahnbildung nach Art einer Zwangsneurosen) que se caracteriza por su fugacidad y sus reincidencias, y por último la neurosis de referencia, que abarca todos aquellos estados "en que el valor de realidad concedido a las ideas de relación permanece más acá de los límites asignados a la psicosis". Estos estados son, en suma, las formas atenuadas del delirio, frecuentes en las formas más ligeras (por ejemplo en el grupo del llamado delirio de los masturbadores), y sobre todo en las secuelas secundarias que suele dejar el delirio ${ }^{4}$.

La evolución, dice Kretschmer, confirma la psicogenia de la enfermedad. "Esta evolución es relativamente favorable". Las psicosis ligeras no suelen caer en las manos del médico de asilo, sino en las del médico de consultorio particular. Tratadas por él en tiempo oportuno, tienden a desaparecer completamente, dejando una corrección completa del delirio ${ }^{4}$.

De ciertas formas, como el delirio de los masturbadores, incluso después de manifestaciones graves, parece que puede decirse que son completamente curables ${ }^{4}$. 
En los casos que están a medio camino, "la concepción delirante pasa al segundo plano sin que aparezca, no obstante, la consciencia de la enfermedad".

Por último, incluso en las psicosis sensitivas que han mostrado manifestaciones graves de confusión aguda, no hay que desesperar. Tres casos de esta índole, observados por Kretschmer, culminaron, después de una evolución que duró de tres a seis años más o menos, en una neurosis de referencia, resultado que se puede considerar como favorable, si se compara la gravedad de los síntomas en la fase de estado, permitiendo la reanudación de la actividad profesional ${ }^{4}$.

\section{LA REACCIÓN PRIMITIVA DE REFERENCIA DE KURT SCHNEIDER}

K. Schneider, en el capítulo tercero de su patopsicología clínica, en el que describen las reacciones vivenciales anormales, comenta que en ciertos casos muy raros existen reacciones deliroides a consecuencia del pánico agudo ante una inmediata amenaza por parte de otra persona: una comprensión y una interpretación falsas e insensatas de algo inocuo que es captado en un sentido amenazador. Pudiendo surgir también equivocaciones sensoriales en forma de ilusiones ${ }^{9}$.

El autor ilustra este tipo de reacciones con el siguiente caso clínico: "Un robusto e ingenuo aldeano de veinticuatro años, procedente de un pequeño pueblecillo de la Baja Baviera y que jamás estuvo en una gran ciudad, llega a Colonia con el fin de visitar a su novia. A poco de llegar se cree ya observado por la gente, y de noche, en el asilo donde se aloja, cree también que dos compañeros de habitación le amenazan. Preso del máximo temor y huyendo de sus supuestos perseguidores corre a través de la ciudad para refugiarse finalmente en el jardín de una villa. Es descubierto y detenido como ladrón por la policía. Comienza en seguida una furiosa lucha contra los funcionarios del puesto de policía y de la cárcel, en los que cree reconocer a gentes del asilo que se han disfrazado. En total hiere de bastante consideración a siete empleados. También oye hablar en su celda: sus padres han sido asesinados y él debe asimismo morir. Transcurridos dos días se tranquiliza, y pronto vuelve a su juicio, explicándose asimismo todo lo sucedido como debido a su miedo. El recuerdo de los dos días no carece de lagunas. Una catamnesis realizada dos años más tarde revela que durante todo este tiempo no ha vuelto a recaer" .
"Designadas con anterioridad tales reacciones como "delirio primitivo de referencia", correspondiente a la reacción primitiva de Kretschmer: un reaccionar casi reflejo realizado antes aún de haber comprendido bien la vivencia, o antes incluso de haberla elaborado. Para reaccionar así no se precisa de una personalidad primitiva, sino que cualquiera es susceptible de ello, dada una determinada situación. No sucede, pues, como en el "delirio sensitivo de referencia", el cual presupone una personalidad específica. No se trata, sin embargo, de un auténtico delirio, de una percepción delirante inmotivada, por lo cual resulta preferible hablar de reacción primitiva de referencia. Precisamente las falsas interpretaciones poseen un motivo: la espera angustiosa. Dichos estados ceden siempre con bastante rapidez a una conversación y explicación aclaratorias y quedan reemplazados por una completa cordura y por la corrección de los errores sufridos"

K. Schneider considera importante el papel desempeñado por la personalidad en las reacciones vivenciales, pues es siempre la personalidad lo que reacciona a las vivencias, concluyendo que cuanto más insignificante sea el motivo que despierta la reacción, tanto en magnitud, aspecto o duración, tanta mayor importancia ha de otorgarse al papel desempeñado por la personalidad de base $^{9}$. Añadiendo que las reacciones a conflictos íntimos se hallan vinculadas a personalidades muy determinadas y desde luego, casi siempre, a sensitivos, inseguros de sí mismos (todo íntimo sentimiento de infelicidad nace de la inseguridad acerca de sí mismo, la confianza en sí mismo no permite que aquél surja, así como permite también liquidar fácilmente las contrariedades procedentes del exterior) ${ }^{9}$.

\section{LAS CLASIFICACIONES INTERNACIONALES}

En 1952, la primera edición del DSM (DSM-I), subdivide las reacciones paranoides en paranoia o trastorno crónico con delirio sistematizado y los estados paranoides, cuadros de patología más aguda con delirios menos sistematizados ${ }^{10}$.

La segunda versión (DSM-II) publicada en 1968 conserva estas nociones ${ }^{10}$.

La tercera (DSM-III), de 1980, incluye en los trastornos paranoides la paranoia, término con el que se sigue indicando la forma crónica y los trastornos paranoides agudos y atípicos para formas de mejor evolución ${ }^{10}$.

A partir de la DSM-III, [el DSM-III-R (1987), DSM-IV (1994), DSM-IV-TR (2000)], se modifica el término 
paranoia y paranoico llamando a estos trastornos delirantes e incluyendo en los mismos los siguientes tipos: persecución, grandeza, celos, erotomanía, y sómatico, no incluyendo ninguno de los delirios de los clásicos: el delirio sensitivo de Kretschmer, delirios de interpretación y reivindicación de Serieux y Capgras, delirios imaginativos de Dupré y los delirios querulantes.

\section{CURSO Y PRONÓSTICO DE LA PARANOIA SEGÚN LOS ESTUDIOS ACTUALES}

El curso del trastorno delirante (paranoia) es muy variable, pudiendo existir remisiones completas o pudiendo evolucionar hacia formas crónicas ${ }^{10}$.

Gran parte de la información acerca de la evolución del trastorno delirante deriva de las investigaciones de Reterson (1991 y 1993). Sus estudios de seguimiento revelan que en las formas crónicas el 53\% de los casos presentan remisión, el $10 \%$ se atenúan y el $31 \%$ no se modifican ${ }^{10}$.

En las formas mas agudas, en la mitad de los casos se presenta una remisión duradera, el $37 \%$ recaen y sólo el $10 \%$ se cronifica ${ }^{10}$.

Los estudios de Jorgensen (1994) alumbran que las remisiones se dan entre un tercio y la mitad de los casos, y en cuanto al pronóstico el autor destaca que cuanto mas abrupto y temprano es el comienzo del trastorno delirante, más favorable es el pronóstico. La existencia de factores precipitantes, el buen nivel de adaptación socio-laboral, el ser mujer y el estar casado, implican mayor probabilidad de reversión ${ }^{10}$.

Según el tipo de delirio, el persecutorio es el que más frecuentemente tiene un pronóstico alentador, mientras que el de grandiosidad y el celotípico son los de peor pronóstico ${ }^{10}$.

\section{REFLEXIONES DE LA AUTORA}

Es evidente que, dentro de los trastornos delirantes en la práctica clínica, hay formas de evolución benigna, pero es importante remarcar que estas formas benignas se asocian a personalidades de base con rasgos de tipo neurótico: personalidades con rasgos obsesivos, depresivos, evitativos, dependientes, histriónicos o una mezcla mixta de los mismos.

Cuando la personalidad de base que subyace al Delirio es de tipo narcisista o paranoide, o mezcla de ambas, la evolución es sombría, ya que estos rasgos se hacen incompatibles con una buena capacidad de introspección y autocrítica, capacidad esencial para el trabajo terapéutico.
Las personalidades sensitivas existen. K. Schneider incluía a los sensitivos de Kretschmer dentro de los inseguros de sí mismo y T. Millon los incluye dentro de los evitativos ${ }^{11}$, sin embargo, los sensitivos descritos por Kretschmer obedecen, a mi parecer, a una combinación de rasgos obsesivos, evitativos, depresivos y dependientes.

De los obsesivos toman la tendencia a la organización, al perfeccionismo y a llevar una vida estructurada. Esta tendencia a cuestionar el mundo en términos de reglas, normas, programaciones y jerarquías les hace rígidos, obstinados e indecisos, con propensión a alterarse ante situaciones nuevas ${ }^{11}$.

Se consideran, a sí mismos, como los obsesivos: aplicados, entregados al trabajo, meticulosos, eficientes y dignos de confianza, valorando en exceso los aspectos del sí mismo que se relacionan con la disciplina, la perfección, la prudencia y la lealtad ${ }^{11}$.

Con los dependientes comparten la expresión afectiva pacífica con calidez, ternura, sin tendencia a la competitividad y evitando tímidamente la tensión social y los conflictos interpersonales. Son devotos de los demás, subordinando en muchas ocasiones sus ideas y opiniones para evitar conflictos y amenazas en el terreno relacional ${ }^{11}$.

Con los evitativos tienen en común esa eterna búsqueda de aceptación, junto al miedo de implicarse, manteniendo la distancia y la privacidad por miedo a sentirse humillados o avergonzados ${ }^{11}$.

De los depresivos nos recuerdan la tendencia a sentirse culpables y cuestionarse la autoestima ${ }^{11}$.

Los trastornos de personalidad sensitivos, como el resto de trastornos de personalidad, pueden asociarse a cualquier trastorno mental del eje I.

En mi dilatada experiencia clínica he encontrado trastornos de personalidad sensitiva tras todo tipo de trastornos (Distimias, T. Ansiedad, T. Bipolares, Esquizofrenias, T. Delirantes) y lo único que puedo añadir a los estudios de los clásicos es que, independientemente de los trastornos que padezcan en el eje I, esa personalidad de base sensitiva suele condicionar una buena evolución, ya que son pacientes empáticos, cumplidores y con buena adherencia, factores que fomentan la alianza terapéutica. 


\section{BIBLIOGRAFÍA}

1. Wernicke C. Zirkumskripte Autopsychose aufgrund einer überwertigen Idee. Dtsch med Wschr. 1892; 18:581-82. En: Kretschmer E. El delirio sensitivo de referencia. Madrid: Triacastela; 2000.

2. Friedmann "Beiträge zur Lehre von der Paranoia". Mschr $f$ Psychiat. 1905; 17:467. En: Lacan J. De la Psicosis paranoica en sus relaciones con la personalidad. Madrid: Siglo veintiuno editores; 2006.

3. Gaupp. R. "Über paranoische Veranlagung und abortive Paranoia". Allg Zschrf Psychictr. 1910;317. En: Lacan J. De la Psicosis paranoica en sus relaciones con la personalidad. Madrid: Siglo veintiuno editores; 2006.

4. Lacan J. De la Psicosis paranoica en sus relaciones con la personalidad. Madrid. Siglo veintiuno editores, 2006.

5. Kraepelin E. Lehrbuch p. 1723; En Lacan J. De la Psicosis paranoica en sus relaciones con la personalidad. Madrid: Siglo veintiuno editores; 2006.

6. Lange. "Über die Paranoia und die paranoische Veranlagung". Zschr Ges Neurol Psychiatr. 1924; 94:85-152. En: Lacan J. De la Psicosis paranoica en sus relaciones con la personalidad. Madrid: Siglo veintiuno editores; 2006.

7. Kretschmer E. El delirio sensitivo de referencia. Madrid: Triacastela; 2000.

8. Ey H. Tratado de psiquiatría. Barcelona: Toray- Masson S.A.; 1965.

9. Schneider K. Patosicología clínica. Madrid: Paz Montalvo; 1975.

10. De Portugal E. and Cervilla J. Trastorno delirante. Madrid: Aula Médica; 2004.

11. Millon T. Trastornos de la personalidad. Más allá de la DSMIV. Barcelona: Masson; 1996. 